\title{
Construction of Mathematical Evaluation Model of the Countries' Sustainable Development Degree
}

\author{
Yazhu Wang \\ North China Electric Power University, Baoding 071000, China \\ 1481030346@qq.com
}

Keywords: AHP; CEI; Cl; SI; Sensitivity analysis.

\begin{abstract}
In order to study the sustainable development of a country, we establish a model to evaluate its sustainable development degree. Before modeling, 20 indicators are selected from three aspects of social development, economic growth and resource environment. Then the cluster is applied to examine the index set's redundancy. We construct three sub models to measure three sides of a country's sustainable development degree. Comprehensive Evaluation Index Model (CEIM): CEI shows development foundation, figured out by using AHP. Sustainable Index Model (SIM): SI is defined to express the keeping growing ability. Coordination Index Model (CIM): CI is defined to represent the ability of coordinated development. Finally, we analyze the sensitiveness for the weights calculated by AHP, drawing the conclusion that our model is insensitive to the change of subjective factors.
\end{abstract}

\section{Background and Significance of The Research}

Sustainable development is defined by the 1987 Brundtland Report [1]. Since its conception, sustainable development has become a goal for all the countries. Thus, it's of vital significance to measure the sustainable development degree of a country. A mathematical model to evaluate the sustainable development degree of a country is established in the thesis. And it could be used to provide guidance for the country to formulate its sustainable development strategy.

\section{Construction of Index Set}

Take Lao as an example, 20 indicators [5] are selected from three aspects of social development, economic growth and resource environment. In order to test the rationality of the selected indicators, the cluster is applied to examine the index set's redundancy [3].

We could figure out the correlation coefficient matrix of all the data after transformation by using MATLAB. Cluster the indicators by means of cluster analysis. The redundancy rate is defined as follows:

$\lambda=\frac{n}{m} \times 100 \%$

$\lambda$ represents redundancy rate;

$\mathrm{N}$ represents the number of redundancy indexes;

$M$ represents the number of all indexes;

\section{Construction of The Model}

We set up three sub models to evaluate sustainable development degree of a country from different angles, they are: CEIM, SIM, CIM [6].

\subsection{Sub Model 1-Evaluation Index Model}

Sustainable development is a very complex research object. The construct of the index set is equivalent to make hierarchical processing of it. After acquiring the data of each level, as long as the index weight is confirmed, we will figure out the result of comprehensive evaluation [2]. Obviously, the method of AHP [4] is quite suitable for this problem. 
The comprehensive evaluation index $C E I$ is determined by the following formula:

$$
C E I=\sum \omega_{i} \times D_{i}
$$

$D_{i}$ represents the value of the indexes after normalizing.

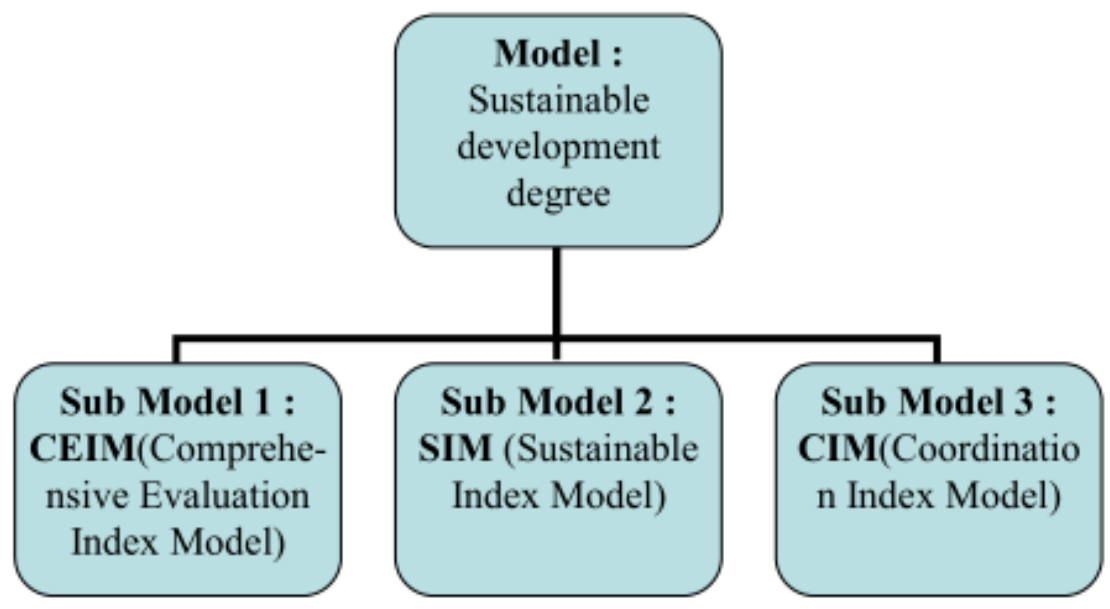

Figure 1 Model decomposition

\subsection{Sub Model 2-Sustainable Index Model}

One of the basic characteristics of sustainable development is when its progress is close to sustainability, the greater the change rates of social development, economic growth and resources\&environment indexes, the stronger sustainable ability the country will hold; If the change rate is slow, then we hold the opinion that the sustainable development ability of the country is weak. If the development rate is a negative number, we think that it's unsustainable. Thus we introduced the sustainable index to measure the speed of country factors in which they are becoming sustainable.

According to the characteristics of national development, the basic elements of national development consists of social factors, economic factors and resources\&environment factors. And they could be measured by social development index, economic growth index, resources\&environment index respectively. The value of all the indicators ranges between 0 and 1 after normalizing, that is to say all the indicators changes between the poorest state (Its value is 0 ) and the best condition (Its value is 1). The national sustainable development index $S I$ is defined by the following formula:

$$
\begin{aligned}
& \begin{cases}S I=\frac{X_{2}-X_{1}}{1.732-X_{1}} & X_{2}>X_{1} \\
\frac{X_{2}-X_{1}}{X_{1}} & X_{2}<X_{1} \\
0 & X_{2}<X_{1}\end{cases} \\
& \text { s.t. } \begin{cases}X_{1}=\sqrt{B_{11}^{2}+B_{21}^{2}+B_{31}^{2}} \\
X_{2}=\sqrt{B_{12}^{2}+B_{22}^{2}+B_{32}^{2}}\end{cases}
\end{aligned}
$$

Note: SI represents sustainable index, B11 and B12 represent social development indexes in different period. B21 and B22 represent economic growth indexes in different period. B31 and B32 represent resources\&environment indexes in different period.

\subsection{Sub Model 3-Coordination Index Model}

According to the connotation of sustainable development, we know one of the basic features of country sustainable development is the coordination of society, economy and resources\&environment. To study sustainable development quantificationally, we introduced the coordinating index $C I$ to evaluate the coordinate development of society, economy and 
resources\&environment. The mathematical intention is the speed balance of social, economic, resources\&environmental development. Any side is partial, the comprehensive benefit will be reduced. The national sustainable development index $C I$ is shown as follows:

$$
\begin{gathered}
C I=\frac{X+Y+Z}{\sqrt{X^{2}+Y^{2}+Z^{2}}} \\
\text { s.t. }\left\{\begin{array}{l}
X=B_{12}-B_{11} \\
Y=B_{22}-B_{21} \\
Z=B_{32}-B_{31}
\end{array}\right.
\end{gathered}
$$

Note: CI represents coordination index.

\section{Sensitivity Analysis}

There exists the influence of subjective factors in the process of constructing comparison matrix through the AHP method, so we will conduct sensitivity analysis with the weights, take the weight that rule layer to target layer as an example.

We assume that the error $\Delta= \pm 1$ in matrix assignment. Then we could get different matrixes in different assignment process, and obtain different weights of all the indicators. We test 5 times and get the following results as Figure2 shows;

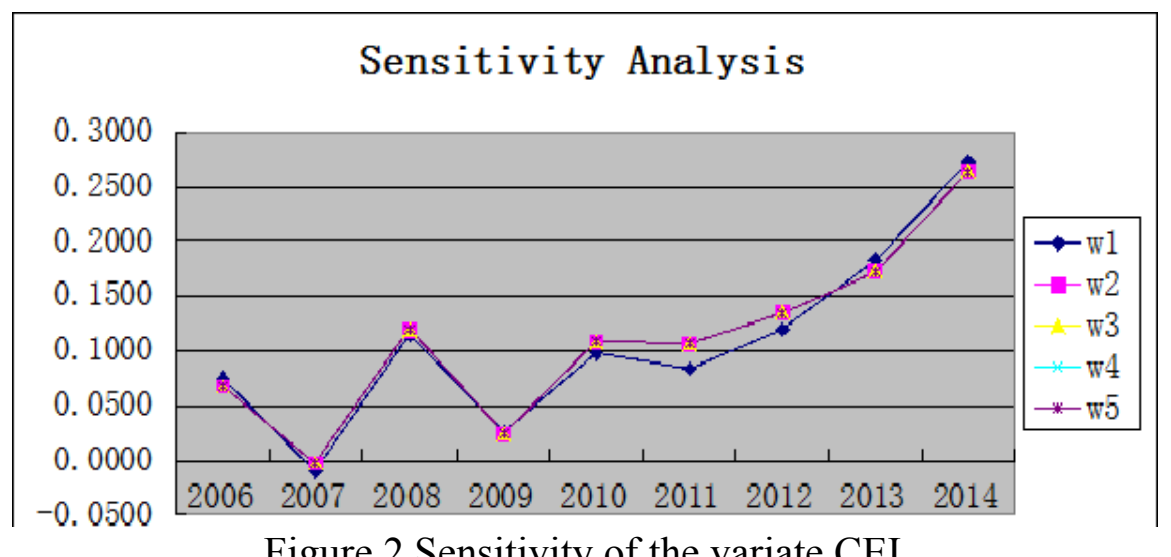

Figure 2 Sensitivity of the variate CEI

\section{Summary}

The establishment of the model is based on the definition and connotation of sustainable development. Therefore we have adequate theoretical basis, which become the foundation of model's suitability and effectiveness

For the determination of index weight in the model, we use the AHP which is very convenient and effective in complex problems. While, the influence of subjective factors couldn't be ignored in AHP, which are easy to cause that the index weight distribution is not reasonable.

\section{References}

[1] World Commission on Environment and Development (WCED). 1987. Our Common Future. New York: Oxford University Press, 1987, 8.

[2] Lv Minglun, Liu Weiguo. Theory of regional sustainable development [J]. Geographical research, 1998, 17(2):131-137.

[3] Fu Bojie, Chen Liding, Ma Cheng. Sustainable land use evaluation index system and method [J].Journal of natural resources, 1997, 12(2), 113-118. 
[4] Jiang Qiyuan, Xie Jinxing, Ye Jun. Mathematical Modeling. Higher Education Press. 2005.5, p. 224-244.

[5] He Lijun, Gao Bo. The establishment of the comprehensive evaluation index system of regional sustainable development at the county level [J]. Journal of Hebei University of technology, 2006, 35(4):20-23.

[6] Teng Yunxian. Study on Index System of Regional Sustainable Development Based on Analytic Hierarchy Process Method. 2009.5. 